\title{
Editorial
}

\section{Fractional Differential Equations 2011}

\author{
Fawang Liu, ${ }^{1}$ Om P. Agrawal, ${ }^{2}$ Shaher Momani, ${ }^{3}$ \\ Nikolai N. Leonenko, ${ }^{4}$ and Wen Chen ${ }^{5}$ \\ ${ }^{1}$ Mathematical Sciences, Queensland University of Technology, GPO Box 2434, Brisbane, \\ QLD 4001, Australia \\ 2 Department of Mechanical Engineering and Energy Processes, Southern Illinois University, \\ Carbondale, IL 62901, USA \\ ${ }^{3}$ Department of Mathematics, The University of Jordan, Amman 11942, Jordan \\ ${ }^{4}$ School of Mathematics, Cardiff University, Cardiff CF24 4AG, UK \\ ${ }^{5}$ Department of Engineering Mechanics, Hohai University, Nanjing 210098, China
}

Correspondence should be addressed to Fawang Liu, f.liu@qut.edu.au

Received 29 November 2011; Accepted 29 November 2011

Copyright (C) 2011 Fawang Liu et al. This is an open access article distributed under the Creative Commons Attribution License, which permits unrestricted use, distribution, and reproduction in any medium, provided the original work is properly cited.

It is our pleasure to bring this special issue of the International Journal of Differential Equations dedicated to fractional differential equations (FDEs).

In recent years, a growing number of work by many authors from various fields of science and engineering deal with dynamical systems described by fractional partial differential equations. Due to the extensive applications of FDEs in engineering and science, research in this area has grown significantly all around the world.

This special issue of Fractional Differential Equations consists of 13 original articles covering various aspects of FDEs and their applications by some of the prominent researchers in the field. These papers could be broadly grouped into three categories, namely, numerical and approximate schemes to solve fractional dynamical models (1st to 5th paper), existence and uniqueness of solutions of fractional differential equations and other theoretical results (6th to 11th paper), and application of fractional differential equations in various fields (12th and 13th paper). Other papers could also have been considered in this last category. However, because of their emphasis, they have been included in the first two categories.

The first paper introduces a new modified step variational iteration method for solving biochemical reaction model. The second and third papers use homotopy analysis method for solving a space- and time-fractional foam drainage equation and nonlinear coupled equations with parameters derivative, respectively. The fourth paper develops a new application of Mittag-Leffler function method and extends the application of the method to fractional linear differential equations. The fifth paper proposes an explicit numerical method for the 
fractional Cable equation together with a stability and convergence analysis of the numerical method by means of a kind of von Neumann method.

The sixth paper investigates Malliavin calculus of Bismut type for fractional powers of Laplacians in semigroup theory. The seventh paper derives a sufficient condition on asymptotical stability of nonlinear fractional differential system with Caputo derivative. The eighth paper studies existence and uniqueness theorem of fractional mixed VolterraFredholm integro-differential equation with integral boundary conditions. The ninth paper proves the existence of positive solution for fractional differential equation with nonlocal boundary consider. The tenth paper gives existence of solutions for a nonlinear fractional multipoint boundary value problem at resonance. The eleventh paper proves the uniqueness of the Gellerstedt problem by energy integral method and the existence by reducing it to the ordinary differential equations.

The twelfth paper studies slip effects on fractional viscoelastic fluids. The final paper addresses antisynchronization phenomena in nonidentical fractional-order chaotic systems using active control.

Thus, this special issue provides a wide spectrum of current research in the area of FDEs, and we hope that experts in this and related fields would find it useful.

Fawang Liu

Om P. Agrawal

Shaher Momani

Nikolai N. Leonenko

Wen Chen 


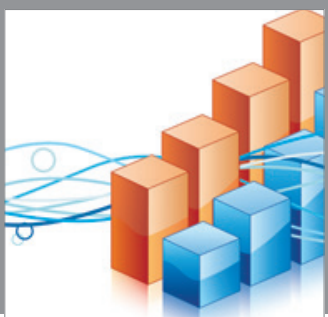

Advances in

Operations Research

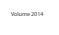

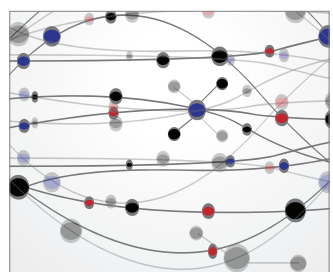

\section{The Scientific} World Journal
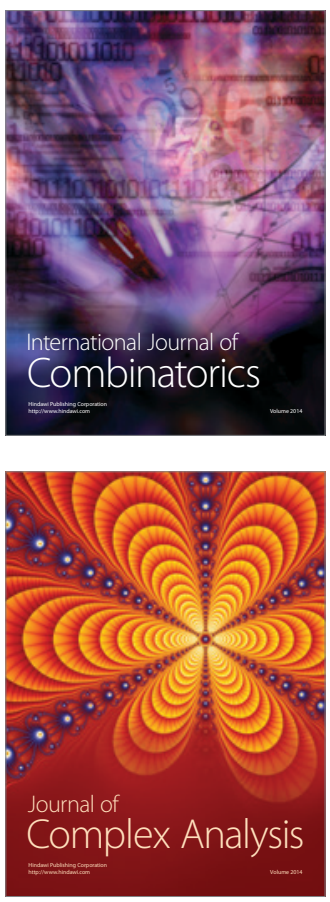

International Journal of

Mathematics and

Mathematical

Sciences
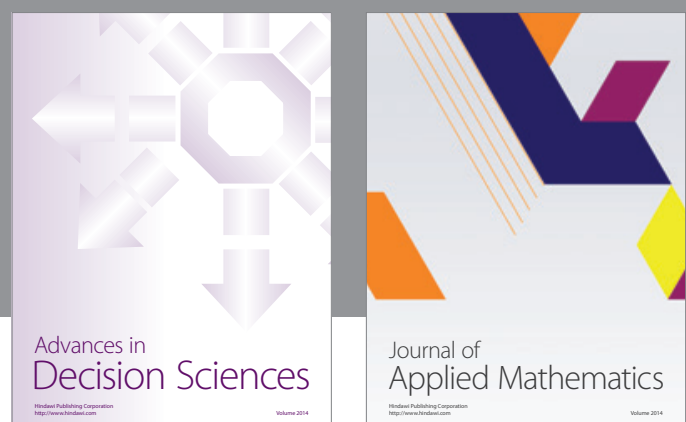

Journal of

Applied Mathematics
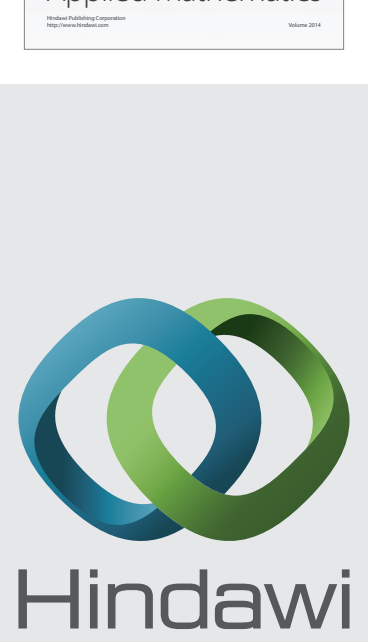

Submit your manuscripts at http://www.hindawi.com
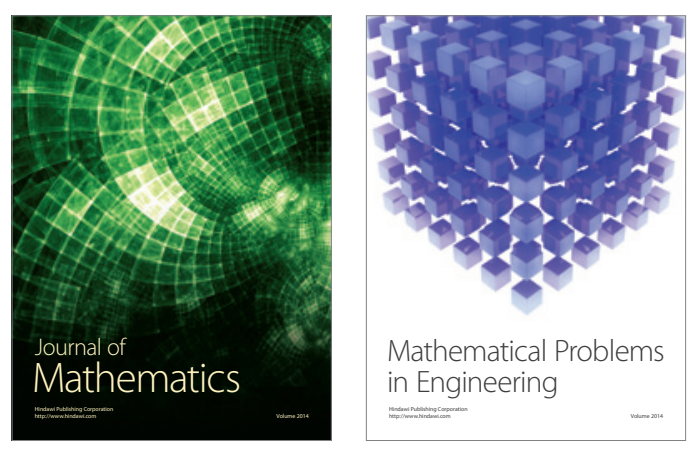

Mathematical Problems in Engineering
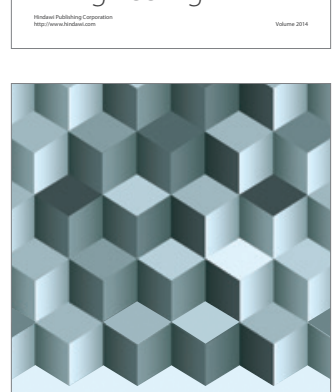

Journal of

Function Spaces
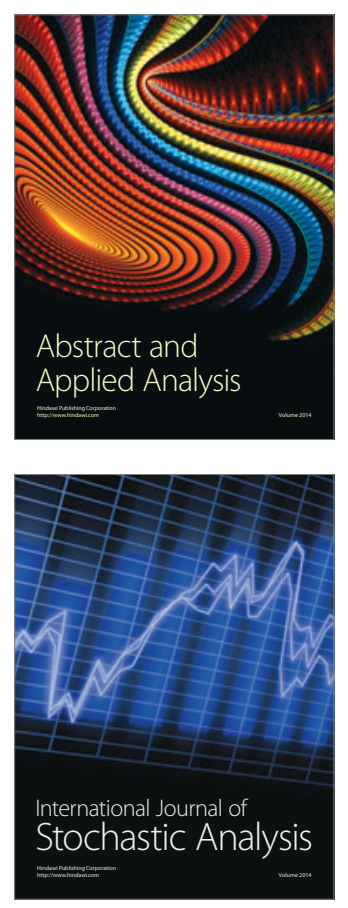

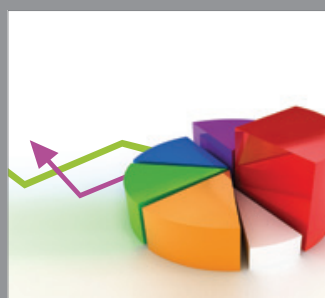

ournal of

Probability and Statistics

Promensencen
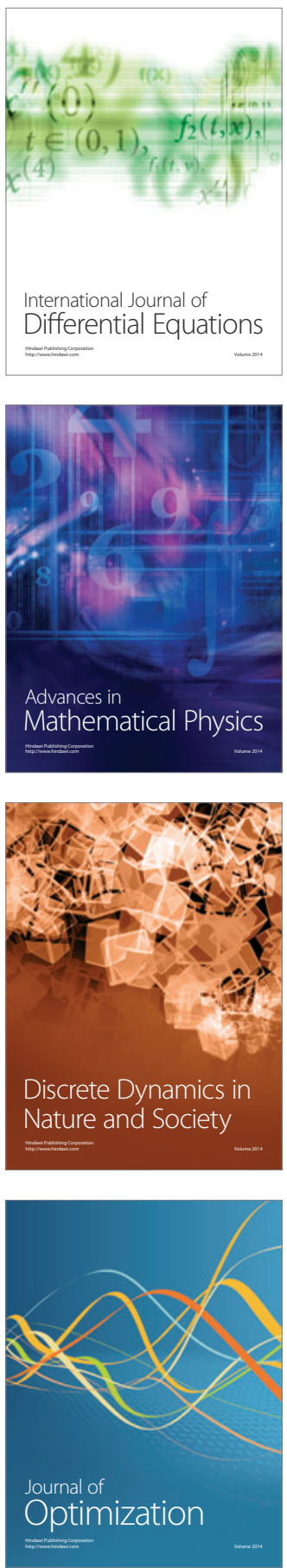